\title{
Effect of size of copper nanoparticles on its catalytic behaviour in Ullman reaction
}

\author{
MOHD. SAMIM, N K KAUSHIK and AMARNATH MAITRA* \\ Department of Chemistry, University of Delhi, Delhi 110 007, India
}

MS received 9 April 2007; revised 28 May 2007

\begin{abstract}
The condensation of iodobenzene to biphenyl is an industrially important reaction due to its significant role in organic synthesis as drug intermediates. The reaction takes place in the presence of copper powder as catalyst. We have shown in this paper that the size of the copper nanoparticles as well as its exposed surface area is responsible for the yield of chemical reaction. The uncapped copper powder showed a 43\% conversion of iodobenzene to biphenyl in $5 \mathrm{~h}$ under our experimental conditions. Same amount of copper nanoparticles (size, $\sim 66 \mathrm{~nm}$ diameter) prepared by citrate capping showed $88 \%$ conversion of iodobenzene to biphenyl, which increased to about $95 \%$ when $8 \mathrm{~nm}$ diameter capped copper nanoparticles are used. Surprisingly, $5 \mathrm{~nm}$ size copper nanoparticles showed no change in the yield of about $95 \%$.
\end{abstract}

Keywords. Ullman reaction; copper nanoparticles; biphenyl; size dependent catalytic behaviour.

\section{Introduction}

A burst of research activity is witnessed in recent years in the area of synthesis and fabrication of different size and shape of metal nanoparticles. Nanometer sized particles display many interesting optical, electronic, magnetic and chemical properties yielding applications in biological nanosensors, optoelectronics, nanodevices, nanoelectronics, information storage and catalysis (Feldheim et al 2002). Amongst many metals like $\mathrm{Au}, \mathrm{Ag}, \mathrm{Pd}, \mathrm{Pt}$, towards which research is directed, copper and copper based compounds are the most important materials. The metallic $\mathrm{Cu}$ plays a significant role in modern electronics circuits due to its excellent electrical conductivity and low cost nanoparticles (Schaper et al 2004). So $\mathrm{Cu}$ will gain increasing importance as is expected to be an essential component in the future nanodevices due to its excellent conductivity as well as good biocompatibility and its surface enhanced Raman scattering (SERS) activity (Pergolese et al 2006). Metallic copper nanocrystals homogeneously dispersed in silica layers have attracted great attention recently for the development of nonlinear optical devices (Flytzanis et al 2005). Such composite materials offer exciting possibilities of potential thin films device applications with novel function arising from size quantization effect. In the light of fast and growing applications of metallic copper nanoparticles, a reproductive method of synthesis with a specific size, well defined surface composition, isolable and redispersable properties remains a

*Author for correspondence (maitraan@yahoo.co.in) challenging task to a synthetic chemist. The ability to scale up the synthesis to bulk scale will gain increasing importance as more applications are established. However, most of the synthetic methods either yielded particles of irregular shape with wide size distribution and required high temperature and pressure condition or produced particles with reduced catalytic activity and inability to reuse the particles. Very little work has been carried out on the cold synthesis of copper nanoparticles (Zhu et al 2005). In view of the above limitations we have attempted the synthesis of size-controlled copper nanoparticles through a simple one-phase aqueous route using citrate as capping agent and used these nanoparticles as catalyst for Ullmann type coupling reactions of aryl iodide (Hassan et al 2002).

In this paper, we have reported the synthesis of copper nanoparticles by reducing the copper ions with sodium borohydride and in situ capping the metallic nanoparticles with citrate ions. We have varied the particle size by modulating the concentrations of reactants and capping agent. The catalytic activities of these particles of different sizes have been tested on the yield of production of biphenyl from iodobenzene in Ullman reaction.

\section{Experimental}

Copper nanoparticles were prepared in aqueous solution by reducing $\mathrm{Cu}^{2+}$ ions with sodium borohydride. The metallic copper produced was immediately capped by sodium citrate to prevent further growth. The detailed synthetic procedure of copper nanoparticles was as follows: The particles were prepared in aqueous phase by chemical 
reduction of cupric salt solution using sodium borohydride in the presence of capping agent. In a typical set, $10 \mathrm{ml}$ $\mathrm{CuSO}_{4}$ solution $(0 \cdot 0125-0 \cdot 075 \mathrm{M})$ was purged with $\mathrm{N}_{2}$ gas for $10 \mathrm{~min}$ to remove the dissolved oxygen and $10 \mathrm{ml}$ of sodium citrate solution $(0 \cdot 2-0.5 \mathrm{M})$ was added and allowed to stir for $30 \mathrm{~min}$ under $\mathrm{N}_{2}$ atmosphere. $1 \mathrm{ml}$ of aqueous solution of sodium borohydride $(0 \cdot 1 \mathrm{M})$ was then added dropwise to it under constant stirring in nitrogen atmosphere. The solution colour changed to dark brown on complete addition of reducing agent indicating the formation of citrate protected copper nanoparticles. The particles were, then, extracted by ultracentrifugation of resultant solution at $30,000 \mathrm{rpm}$ for $1 \mathrm{~h}$. The pelleted nanoparticles were washed repeatedly with ice-cold water to remove any trace of free sodium citrate.

Similar procedure was followed for reduction of cupric ion solution without using capping agent (citrate ion), which shows immediate precipitation of metallic copper indicating formation of macrosize copper particles.

Iodobenzene was purchased from Spectrochem (India) Ltd. The copper powder prepared by the above method was used for catalytic testing without further activation. In a typical reaction, $50 \mathrm{mg}$ of copper particles were added to $1 \mathrm{ml}$ of DMSO solution containing $100 \mathrm{mg}$ of iodobenzene and the mixture was refluxed at $200^{\circ} \mathrm{C}$ for $5 \mathrm{~h}$. After the required time period the reaction system was cooled rapidly to room temperature and the product was separated by thoroughly mixing with $2 \mathrm{ml}$ of saturated ammonium chloride followed by $2.5 \mathrm{ml}$ of dichloromethane which separated two layers with the upper layer containing the product dissolved in dichloromethane. The product was extracted three times by solvent evaporation method to get the pure compound.

The particle size was determined by transmission electron microscopy (TEM) using a JEOL Model JEM $2000 \mathrm{E} \times 200$ electron microscope. Samples were prepared by placing small drops of dispersed particles in water on formvar coated copper grids and allowing the solvent to slowly evaporate at room temperature.

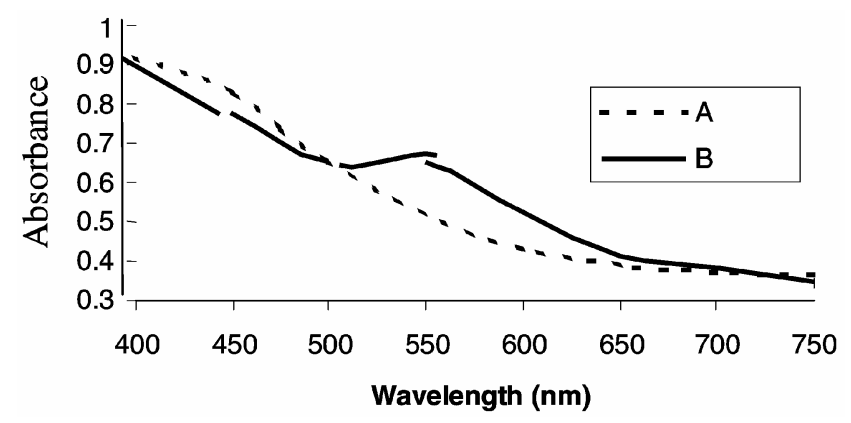

Figure 1. Absorption spectra of $\mathrm{Cu}$ nanoparticles. (A) Smaller size $\mathrm{Cu}$ nanoparticles $(<12 \mathrm{~nm}$ diameter) showing no SPR absorption band and (B) particles of size, $55 \mathrm{~nm}$ diameter, showing characteristic peak of $\mathrm{Cu}$ nanoparticles at $570 \mathrm{~nm}$.
Powder X-ray diffraction measurements were performed on a Bruker AXS D8 diffractometer using pressed pellets as samples with $\mathrm{K} \alpha$ radiation, $\lambda=1.5418 \AA$.

The crystallite sizes of the particles were determined from the XRD spectrum using the Scherrer equation.

\section{Results and discussion}

Colloidal dispersion of metal exhibits absorption bands or broad regions of absorption in the ultra violet-visible range. These are due to the excitation of plasmon resonance or interband transition and are a characteristic property of the metallic nature of the particle. For example, nanosized $\mathrm{Cu}$ particles typically exhibit a surface plasmon peak at around $560 \mathrm{~nm}$ (Creighton and Eadon 1991). However, as shown in figure 1, for the fresh copper nanoparticles (size, $<5 \mathrm{~nm}$ diameter) synthesized here at low copper ion concentration, the corresponding UV-vis absorption spectrum demonstrated a featureless Mie scattering profile without the appearance of an apparent surface plasmon band. This might, atleast in part, be attributed to the small particle size (Chen and Sommers 2001), while large size copper nanoparticles ( 55 nm diameter) synthesized from higher cupric ion concentration showed a characteristic peak at $570 \mathrm{~nm}$.

Figure 2 shows the XRD pattern of capped copper nanoparticles. The peak positions are consistent with metallic copper (Dhas et al 1998). Sharp peaks of copper are observed which indicates the crystalline nature of the product. A strong peak at $2 \theta=42 \cdot 5^{\circ}$ matches exactly with the earlier reported diffractogram (Li 2004) of crystalline metallic copper. The inset in figure 2 represents the selected area electron diffraction (SAED) of these particles, which reveals only diffusive ring patterns, which are most probably attributed to the small particle size and polycrystalline nature of the particles core. Nevertheless, the diffraction features were consistent with the $f c c$ crystal structure of bulk metallic copper with two brightest rings corresponding to the diffraction planes of (111) and (220).

Well-washed and lyophilized capped $\mathrm{Cu}$ nanoparticles showed characteristic IR peaks (spectra not shown) of citrate ion at $3400 \mathrm{~cm}^{-1}, 1391 \mathrm{~cm}^{-1}$ and $1574 \mathrm{~cm}^{-1}$ corresponding to $\mathrm{OH}$ stretching, $\mathrm{C}=\mathrm{O}$ of the carboxylic acid vibration and $\mathrm{C}-\mathrm{O}$ bending, respectively. These peak positions are not significantly different from those of neat citric acid clearly indicating the presence of weakly adsorbed citrate ion on copper nanoparticles surface. DTA/ TG studies (figure not shown) also support the capping of $\mathrm{Cu}$ nanoparticles. DTA/TG of capped nanoparticles showed weight loss corresponding to citrate ion decomposition at $234.5^{\circ} \mathrm{C}$ indicating capping of citrate ion on copper nanoparticles whereas uncapped copper powder did not show such a kind of weight loss. 


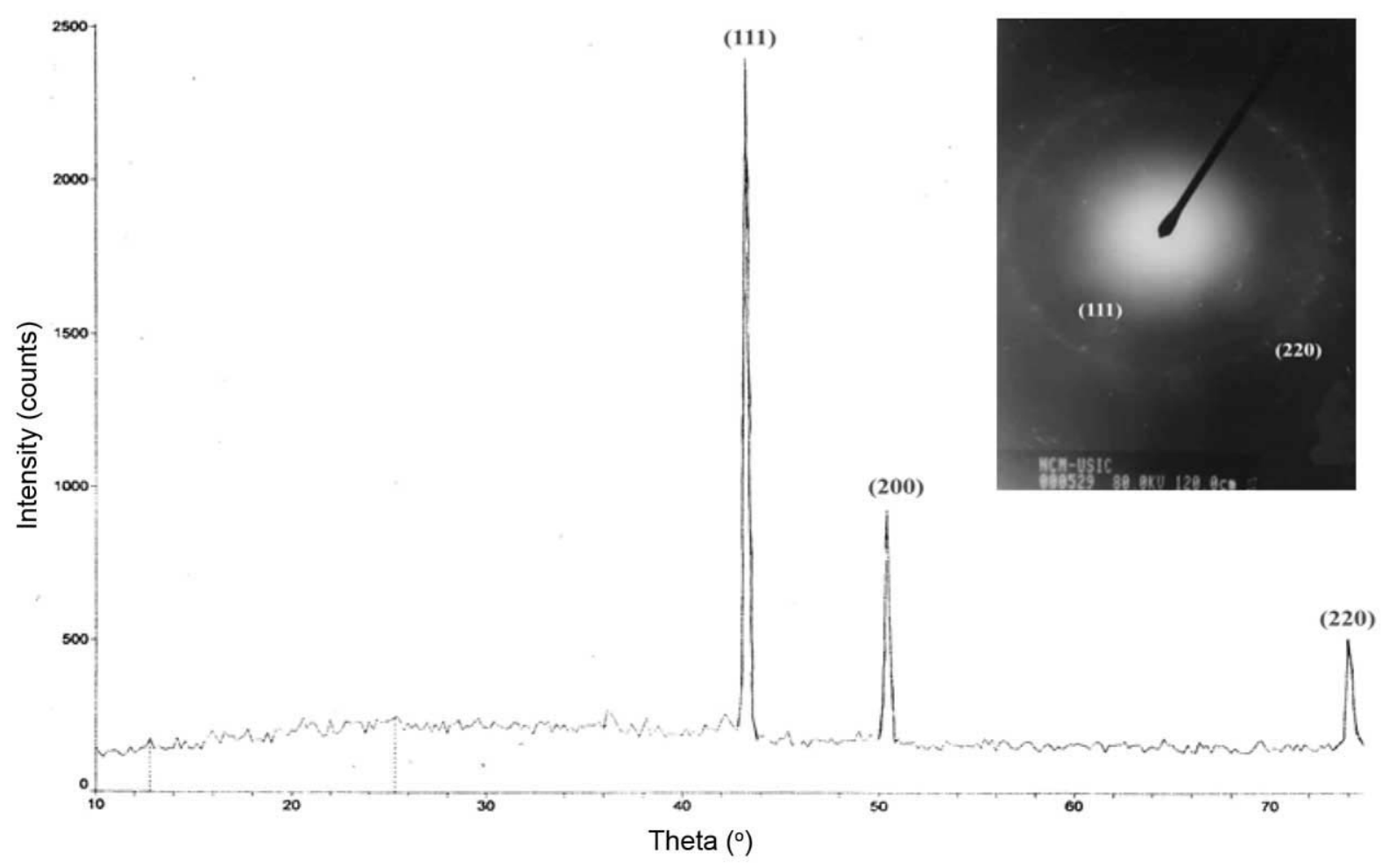

Figure 2. Powder XRD of copper nanoparticles. (Inset: selected area electron diffraction spectrum.)

Table 1. Effect of change of concentration of capping agent (immediate extraction by centrifugation).

\begin{tabular}{lcc}
\hline $\begin{array}{l}\text { Concentration of } \\
\text { added } \mathrm{CuSO}_{4}(\mathrm{M})\end{array}$ & $\begin{array}{c}\text { Concentration of added } \\
\text { capping agent }(\mathrm{M})\end{array}$ & $\begin{array}{c}\text { Size of the } \\
\text { particles }(\mathrm{nm})\end{array}$ \\
\hline 0.0125 & $0 \cdot 5$ & 5 \\
0.0125 & $0 \cdot 3$ & 8 \\
0.0125 & $0 \cdot 25$ & - \\
0.0125 & $0 \cdot 2$ & 12 \\
\hline
\end{tabular}

Table 2. Effect of digestion of the particles in aqueous dispersion for $48 \mathrm{~h}$.

\begin{tabular}{lcc}
\hline $\begin{array}{l}\text { Conc. of added } \\
\mathrm{Cu} \text { ion }(\mathrm{M})\end{array}$ & $\begin{array}{c}\text { Conc. of added } \\
\text { capping agent }(\mathrm{M})\end{array}$ & $\begin{array}{c}\text { Size of } \\
\mathrm{Cu}(\mathrm{nm})\end{array}$ \\
\hline 0.0125 & 0.5 & $33 \cdot 33$ \\
0.0125 & 0.3 & 55.00 \\
0.0125 & 0.25 & 66.66 \\
0.0125 & 0.2 & $90 \cdot 00$ \\
\hline
\end{tabular}

A representative TEM image and size distribution of $\mathrm{Cu}$ nanoparticles synthesized at varying concentrations of added cupric ion (0.0125-0.075 M) and $0.5 \mathrm{M}$ concentration of added citrate ions are shown in figure 3 . It has been noted that the size of particles increases with the concentration of cupric ion without broadening the polydispersity range. This result implies that controlling the concentration of constituent chemicals can control the size of nanoparticles in aqueous medium.

In addition to these well-dispersed small particles, TEM measurements also show the presence of various very large particles of irregular shape. The chain like features of nearby particles shown in figure $3 \mathrm{c}$ are due to the formation of nanoparticles bilayer structures where the top layer sits at the 2-fold saddle sites between neighbouring particles as observed previously with gold and palladium particles of small size dispersity (Chen et al 2000)

Table 1 shows how increasing concentration of citrate ions can decrease the particle size. Using $10 \mathrm{ml}$ of $0.0125 \mathrm{M}$ copper sulphate in the protocol, the average particle size was found to decrease from $12 \mathrm{~nm}$ diameter to $5 \mathrm{~nm}$ diameter when the added citrate ion concentration was increased from $0 \cdot 2-0.5 \mathrm{M}$. On digesting the particles in aqueous dispersion for a long time, coagulation of particles happened resulting in the formation of bigger particles. Thus, $12 \mathrm{~nm}$ diameter particles dispersed in water aggregated to $90 \mathrm{~nm}$ diameter particles when the aqueous dispersion was stirred for $48 \mathrm{~h}$ (table 2). The change in concentration of cupric ion also had an impact on the size of nanoparticles. It was observed that the particles size increased with increase in concentration of cupric ions (table 3 ).

The condensation of iodobenzene to biphenyl was chosen as the model reaction for catalytic activity due to its significant role in organic synthesis. As shown in figure 4, when 

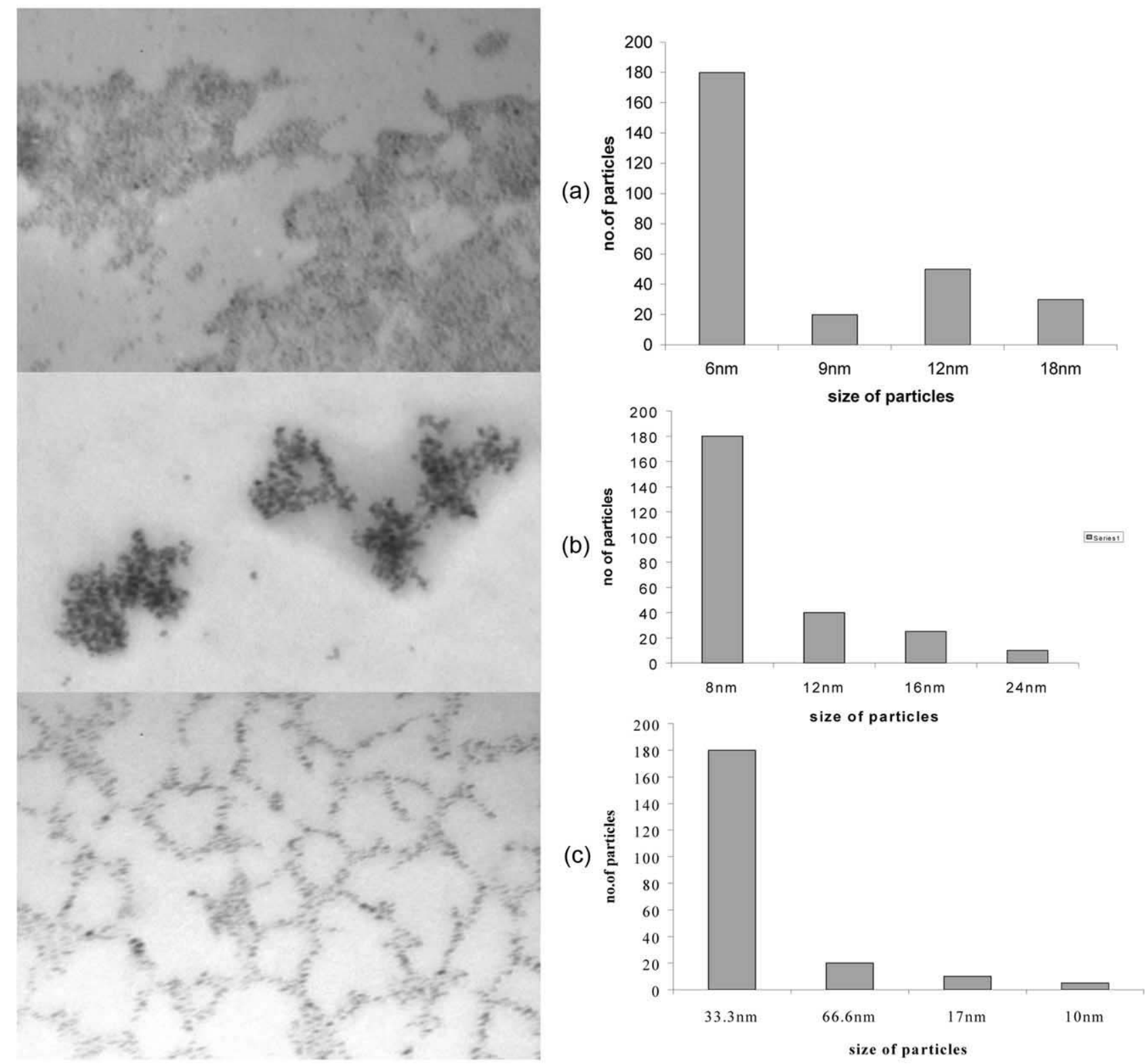

Figure 3. TEM and histogram of copper nanoparticles (capping agent conc. $0.5 \mathrm{M}$ and $\mathrm{Cu}$ ion concentration (a) $0.025 \mathrm{M}$, (b) $0 \cdot 050 \mathrm{M}$ and (c) $0 \cdot 075 \mathrm{M}$ ).

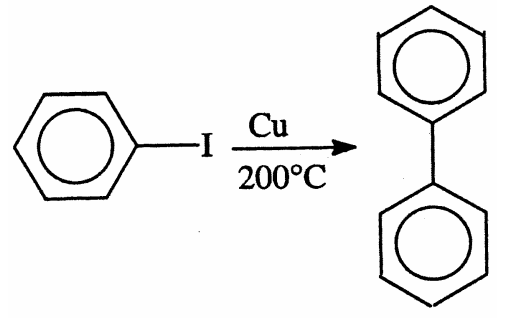

Figure 4. Ullmann reaction in presence of copper catalyst.

iodobenzene is heated at $200^{\circ} \mathrm{C}$ in presence of copper powder it forms diphenyl. Aryl halides couple among themselves to form diaryl derivatives, an important drug intermediate, in presence of copper catalyst.
Table 3. Effect of change of concentration of cupric ion added.

\begin{tabular}{lcc}
\hline $\begin{array}{l}\text { Conc. of added } \\
\mathrm{Cu} \text { ion }(\mathrm{M})\end{array}$ & $\begin{array}{c}\text { Conc. of added } \\
\text { capping agent }(\mathrm{M})\end{array}$ & $\begin{array}{c}\text { Size of } \mathrm{Cu} \\
\text { nanoparticles }(\mathrm{nm})\end{array}$ \\
\hline $0 \cdot 075$ & $0 \cdot 5$ & $33 \cdot 3$ \\
$0 \cdot 050$ & $0 \cdot 5$ & 8 \\
$0 \cdot 025$ & $0 \cdot 5$ & 6 \\
$0 \cdot 0125$ & $0 \cdot 5$ & 5 \\
\hline
\end{tabular}

But unfortunately the yield of the reaction is mostly affected by large size and coating of copper metal by hard material like copper oxide or other strongly adsorbed compounds (Severino and Laine 1983). As a result, the total exposed surface area of the catalyst is significantly 
reduced. This is more true when the nanoparticles are prepared in surfactant solution e.g. in reverse micellar system, the surface is further complicated by the strong adsorption of the surfactant molecules. Some studies have reported heating of the particles at high temperature for removal of adsorbed materials (Bennet et al 1999). This creates additional complication of converting the catalyst surface into irremovable metal oxide, nitride or sulphide. As a result, the catalytic efficiency is drastically reduced.

The total yields of biphenyl in the condensation of iodobenzene for different copper powders are summarized in table 4 . The particle size of different copper powders was included in the table for comparison. The uncapped and undefined macro sized copper powder showed a $43 \%$ conversion of iodobenzene to biphenyl in $5 \mathrm{~h}$ under our experimental conditions. Same amount of copper nanoparticles (size, $\sim 66 \mathrm{~nm}$ diameter) prepared by citrate capping showed $88 \%$ conversion of iodobenzene to biphenyl, which increased to about $95 \%$ when $8 \mathrm{~nm}$ diameter capped copper nanoparticles are used. Surprisingly, $5 \mathrm{~nm}$ size copper nanoparticles showed no change in the yield of about $95 \%$.

The higher catalytic activity towards the condensation of iodobenzene could be due to the higher catalytic surface area of small nanoparticles. It is presumed that the electrophilic nature of the catalyst surface renders a weak bond between the $\pi$-system of benzene ring and vacant $d$ system of copper atoms, which gives slightly positive charge over iodine atom. The whole complex reacts with another molecule of iodobenzene to produce biphenyl and cuprous iodide. The electrophilic nature of copper surface obviates that when the particles are extremely small in size, the electrons are pumped into copper by the benzene $\pi$-system, which usually reduces the band gap between Fermi level and conduction band considerably so that the catalytic activity is also expected to be reduced (Huynh et al 2003). However, copper has $d$-bands well below the Fermi level and the antibonding stage at the top of the $d$ bands end up below the Fermi level and are filled (Sharma et al 2003; Nilsson et al 2005). As a result, the catalytic activity did not decrease rather it remained constant. Any aggregation of the particles in aqueous dispersion leads to lower efficiency. The freshly prepared and capped $\mathrm{Cu}$ nanoparticles also showed good activity for this Ullmann

Table 4. Dependence of yield of iodobenzene (\%) on size of the copper nanoparticles.

\begin{tabular}{lc}
\hline Particles size (nm) & Approximate yield (\%) \\
\hline 90 & 80 \\
67 & 88 \\
35 & 92 \\
8 & 95 \\
5 & 95 \\
Uncapped copper particles of undefined & 43 \\
large sizes and broad polydispersity & \\
\hline
\end{tabular}

type of condensation indicating that any oxide layer formation on the particle surface perhaps inhibits the catalytic activity. Biaryls are available through coupling of the aryl halide with an excess of copper at elevated temperatures $\left(200^{\circ} \mathrm{C}\right)$. The active species is a copper(I)compound, which undergoes oxidative addition with the second equivalent of halide, followed by reductive elimination and the formation of the aryl-aryl carbon bond.

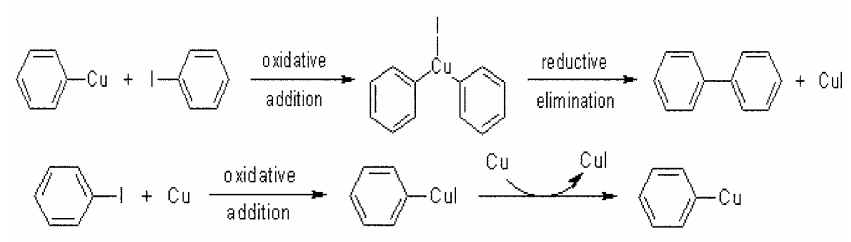

\section{Conclusions}

In this paper, we have described a new synthetic route of preparing copper nanoparticles in aqueous solution at room temperature and coated with a weak adsorbing agent like citric acid on the particle surface which controls the particle size. Up to certain minimum particles size the catalytic efficiency increases below which it remains constant. The method is, however, cost effective and scaling up is possible.

\section{Acknowledgements}

The authors thank Dr N C Mehra, University Science Instrumentation Centre, Delhi University, Delhi, for TEM pictures. One of the authors (ANM) is grateful to the Department of Science and Technology, Government of India, for financial assistance in the form of a research project.

\section{References}

Bennett R A, Stone P and Bowker M 1999 Faraday Discuss 114267

Chen Shaowei and Sommers Jennifer M 2001 J. Phys. Chem. B105 8816

Chen S, Huang K and Sterns J A 2000 Chem. Mater. 12540

Creighton J A and Eadon D G 1991 J. Chem. Soc., Faraday Trans. 873881

Dhas N A, Raj C P and Gedanken A 1998 Chem. Mater. 101446

Feldheim D L and Foss C A (eds) 2002 Metal nanoparticles: Synthesis, characterization and applications (Marcel Dekker Inc.)

Flytzanis C J 2005 Physics B, At. Mol. Opt. Phys. 38 S661

Hassan J, Sevignon M, Gozzi C, Schulz E and Lemaire M 2002 Chem. Rev. 1021359

Huynh Wendy U, Dittmer Janke J, Libby William C, Whiting Gregory L and Alvisatos A Paul 2003 Adv. Funct. Mater. 13 73 
Li C-M, Lei H, Tang Y-J, Luo J-S, Liu W and Chen Z-M 2004 Nanotechnology 151866

Nilsson A, Petterson L G M, Hammer B, Bligaard T, Christensen C H and Norskov J K 2005 Catal. Letts 100 111

Pergolese B, Muniz-Miranda M and Bigotto A 2006 J. Phys. Chem. B110 9241
Schaper A K, Hou H, Greiner A, Schneider R and Philips F 2004 Appl. Phys. A Mater. Sci. Process. 7873

Severino Francisco and Laine Jorge 1983 Indian Eng. Chem. Prod. Res. Dev. 22398

Sharma R K, Sharma P and Maitra A N 2003 J. Colloid. Sci. 265134

Zhu H, Zhang C and Yin Y 2005 Nanotechnology 163079 